\title{
ENDOGENOUS DEPRESSION;
}

\section{TOTAL TRIIODOTHYRONINE (T3) LEVELS IN PATIENTS WITH FIRST EPISODE}

\author{
Dr. Jahangir Sultan, Dr. Tariq Afzal, Dr. Kokab Jabeen
}

ABSTRACT... Objective: The aim of this study to determine the levels of Total triiodothyronine (T3) in patients with first episode of Endogenous depression (prior to medical treatment) and in matched controls. Setting: Punjab Institute of Mental Health. Period: Between Jan. and Dec.2011. Material \& Methods: 100 newly diagnosed depressive patients in the age range of 18-65 years were included in the study. 60 healthy controls were taken from dermatological clinic. Total triiodothyronine (T3) was estimated using enzyme - immuno assay. The results obtained were analyzed using Statistical package of social sciences, Version 7.0 (SPSS 7.0). Results: No significant difference in levels of Total triiodothyronine (T3) was found in depressive patients and control. Conclusions: Lower levels of T3 in depressive patients could lead to subclinical hypothyroidism and refractiveness. Therefore, thyroid profile in refractive patients is suggested.

Key words: Zinc, myocardial infarction, hypozincemia

Article Citation

Sultan J, Afzal T, Jabeen K. Endogenous depression; total triiodothyronine (T3) levels in patients with first episode. Professional Med J Aug 2013;20(4): 595-599.

\section{INTRODUCTION}

A depressive disorder is an illness that involves the body, mood, and thoughts in any given 1 year period and 9.5 percent of population suffers from depressive illness ${ }^{1}$. Depressive illness often interferes with normal functioning and cause pain and suffering to those who care about them. Severe depression can destroy family life as well as the life of the ill person ${ }^{2}$. Depression is often treated as a chronic condition due to frequent relapses that patient endures. This fact high lights the need for adequate treatment duration to prevent a quick relapse of the disorder but also that good follow up and disease education is needed to assist in the early education of future episode ${ }^{3}$.

Predisposing factors include family tendency to depression, genetic, viral, neurological, neurodevelopmemtal, biochemical, psychological abnormalities and structure changes in brain. Depressive disorder affects approximately 18.8 million American adult each year. One in four women and one in 10 men have a serious episode of depression during their life time. Children can also develop depression, which increase the risk of abuse problems and tendency for suicide. Depression is one of the leading causes of disability world-wide ${ }^{4,5,6}$.

World Health Organization (WHO) has predicted that by 2020 , depression will be second leading cause of disability in the world after coronary heart disease. ${ }^{5}$ In present years, researches have shown that physical changes in the body can be accompanied by mental changes as well. Medical illness such stroke, heart attack, cancer, Parkinson's disease and hormonal disorder can cause endogenous depression, making the sick person apathetic and unwilling to care for his physical need, thus prolong up the recovery period. Also a serious loss, difficult relationship, financial problems, and/or stressful changes in life patterns can trigger a depressive episode. Combination of psychological and environmental factors is involved in the cause of depressive disorder ${ }^{7}$.

Triiodothyronine or T3 is a metabolically active hormone produced by the thyroid and the T3 hormone function is associated with almost all processes in the human body. It is the most powerful hormone produced by the thyroid and its functions are associated with the body temperature, growth and heart rate. In comparison with the other thyroid 
hormone, $\mathrm{T} 4$ or thyroxine, $\mathrm{T} 3$ is produced in a smaller quantity and it contains fewer iodine atoms per molecule. The production of both T3 and T4 are activated by the thyroid stimulating hormone or the TSH. Actually, after the deiodination of T4 in the thyroid, thyroxin is converted to $T 3$, hormone which will inhibit THS. Thyroid hormones have a biological half-life up to 2 days and a half. T3 hormone is responsible for increasing the consumption of both oxygen and energy in the body. T3 hormones increase the rate of protein degradation and synthesis. T3 hormones increase as well the rate of glycogen degradation and the one of glucose synthesis. A very important function of T3 hormones is related to the cardiovascular system, they being able to increase the heart rate and its force of contraction. The T3 hormone function is what makes this type of hormone so important for a healthy body.

The relation between thyroid function and depression has long been recognized. Patients with thyroid disorders are more prone to develop depressive symptoms and conversely depression may be accompanied by various subtle thyroid abnormalities. Traditionally, the most commonly documented abnormalities low T3.

The present study was planned to study the T3 hormone levels in patients with first episode of depression and to compare the findings with normal controls.

\section{MATERIAL AND METHOD}

Between Jan. and Dec.2011, 100 newly diagnosed depressive patients in the age range of 18-65 years, having first episode of illness reporting at OPD of Punjab Institute of Mental Health, ( The only facility for psychiatric patients in this part of world starting in 1900, turned into Government Mental Hospital and was renamed Government Hospital for Psychiatric Diseases in 1996. The Hospital was converted to Punjab Institute of Mental Health in 2002 Lahore), and diagnosed as per DCR (Diagnostic Criteria for Research) of ICD-10 (WHO, 1992) were selected. 60 healthy controls were taken from dermatological clinic. A careful history, through physical examination and relevant laboratory investigations were performed to rule at any evidence of endocrinological, hepatic, renal, cardiac or other chronic systemic illness, significant alcoholism or other substance abuse and pregnancy or oral contraceptive use (in case of female subjects).

The depressive patients were rated on Hamilton's depressive rating scale ${ }^{8}$ (Hamilton, 1967) to assess the severity of depression. The controls were matched with the depressive patients with respect to age, sex and socio economic status. The blood sample was drawn in the fasting state between 8 am to 9 am and total triiodothyronine (T3) was estimated using enzyme-immuno assay. The results obtained were analyzed using statistical package of social sciences, version - 7.0 (SPSS-7.0).

\begin{tabular}{|c|c|c|c|c|}
\hline Variable & $\begin{array}{c}\text { Depressive } \\
(\mathbf{N}=100) \\
\text { Mean } \pm 1 \text { 1SD }\end{array}$ & $\begin{array}{c}\text { Control } \\
(\mathbf{N}=60) \\
\text { Mean+1SD }\end{array}$ & t-test & p-value \\
\hline $\begin{array}{c}\text { Total T3 } \\
(\mathrm{ng} / \mathrm{ml})\end{array}$ & $\begin{array}{c}1.299 \pm \\
0.932\end{array}$ & $\begin{array}{c}1.608 \pm \\
0.314\end{array}$ & 1.79 & $\begin{array}{c}\text { Non- } \\
\text { significant }\end{array}$ \\
\hline
\end{tabular}

Table-I. Comparison of total T3 between depressive and the control groups

\section{DISC USSION}

The T3 levels observed in the patients indicate existence of biochemical hypothyroidism. Similar low T3 levels in depression patients have been reported by other scientists ${ }^{10}$. While some have reported low T3 levels in $62 \%$ and $7.6 \%$ respectively ${ }^{11,12}$. Our study indicates low T3 levels in $36.0 \%$ of the patients. Low T3 in higher number of patients in our study could have been due to existence of iodine deficiency reported in this region ${ }^{13,14}$. Our data on T3 in different grades of depression also suggests further aggravation of biochemical hypothyroidism with increase in severity of depression.

Many of the previous studies done in this field had various methodological flaws in the sample selection 
e.g. including the patients having chronic resistant illness or being on psychotropic medication ${ }^{15}$. The present study included only drug-naive patients having first episode of depressive illness. This way, the nonspecific effects of chronicity of illness and pharmacological agents on thyroid functions were taken care off. The most significant finding of the study was low levels of total tri iodothyronine (T3) in the depressive patients in the acute phase of illness as compared to healthy controls ${ }^{16}$. This is in agreement with most of the earlier studies ${ }^{15,16}$. The literature regarding total triiodothyronine (T3) during acute depression is less consistent. In one study it was found marginally elevated T3 during acute depression where as most of the other studies reported either normal or decreased total T3 during acute depression ${ }^{17}$.

Muller and Boning also found elevated thyroxin (T4) and decreased triiodothyronine (T3) during acute depression and concluded that this finding may indicate that there is some defect in the conversion of thyroxin (T4) to triiodothyronine (T3) ${ }^{18}$. We found that the mean value of total triiodothyronine (T3) was lower in depressive patients as compared to controls, though the difference just escaped statistical significance. The decreased triiodothyronine (T3) in depressive patients indicates that the conversion of thyroxin (T4) to triiodothyronine (T3) may be defective in the depressive illness.

Joffe and Levitt found significantly lower total triiodothyronine (T3) in patients with psychotic depression as compared to nonpsychotic depression ${ }^{15}$. Our study found that depressive patients with psychotic features had significantly lower $(p<0.05)$ value of total triiodothyronine (T3) as compared to those without psychotic features. Low values of total triiodothyronine (T3) indicate the presence of subclinical hypothyroidism in depressive patients with the presence of psychotic features as suggested by Joffe and Levitt. Apart from subtle changes in the thyroid functions, many studies have found overt thyroid abnormalities in a minority of depressive patients We found a total of a $4(12 \%)$ patients having grade I and grade II hypothyroidism and 1 patient $(3 \%)$ having frank hyperthyroidism ${ }^{18}$. In the conclusion, we would like to emphasize that this study supports the view that there are subtle but significant abnormalities in the basal level of thyroid hormones in acute depressive illness. There is a need to continue the research efforts in this field to further clarify the aetiopathological significance of altered thyroid functioning in depressive illness. Out of 100 subjects with major psychiatric depression, 6.4\% exhibited low T3 syndrome (mean serum T3 concentration $0.94 \mathrm{nmol} / \mathrm{l}$ vs normal mean serum concentration of $1.77 \mathrm{nmol} / \mathrm{l})$.

\section{CONCLUSIONS}

Our result corroborates the findings of other workers with lower T3 levels in depressive patients and it could lead to subclinical hypothyroidism and refractiveness. Therefore, thyroid profile in refractive patients is suggested. However, preferential conversion of T4 to T3 in these patients is also needed to be explored.

\section{Copyright@ 29 Apr, 2013.}

\section{REFERENCES}

1. Bodie Morey, Kim T. Being there for another with a serious mental illness. Qual Health Res.2009;19(11): 1525-1535.

2. Mary Jan England and Lesli J. Sim. Editors; Committee on Depression, Parenting Practices, and the Healthy Development; National Research Council; Institute of Medicine 2009.

3. Annabel Prce, Lauren Ratan, Ewa Okon-Pocha, Alison Evans Koravangattu Valsraj, J HIGGINSON, Mattew Hotop J Neurosung Psychiatry 2011; 82:914-923 Published on line First: 10 May 2011 do: 1136/tnnp. 2010.23862.

4. Sibillie E. Molecul;ar aging of the brain neuroplasticity and vulnerability to depression and other brain related disorders. Dialogues Clin Neurosci. 2013;15(1):53-65. 3; 55-68. 
5. Hetrick SE, McKenzie JE,Cox GR, Simmons MB, Marry SN. Cochrane database.Sys Rev.2012;11: Cd004851.

6. Carpenter DJ,Fong R,Krause JE,Davis JT,Moore C, Thase ME. Meta-nalysis of efficacy and treatment emergent suicidality in adults by psychiatric indication and age subgroup following initiation of Paroxetine therapy. A complete set of randomized placebo controlled trials.J Clin Psychiatry.2011;72 (11):1503-7.

7. Warden D, Rush AJ, Carmosdy TJ, Kashner Tm, Biggs $\mathrm{MM}$, Crimson ML. Predictors of attrition during one year of depression treatment: A roadmap to personalized intervention. J Psychiatr Pract. 2009;15(2):113-124.

8. $\quad$ Hung Cl, Llucy, Wang SJ. Migraine predicts physical and pain symptoms among psychiatric outpatients. J Affect Disord. 2009;117(1):108-15.

9. Stevens Jones, Medeleine Deville, Debbie Mayes, Fiona Lobban. Self management in bipolar disorders: The syory so far. JMH.2011;20(6)583-592.

10. Strazincky NE, Lambert GW, Masho K, Dawood T. Blunted sympathetic neural response to oral glucose in obese subjects with the insulin-resistant metabolic syndrome. Am J Clin Nut.2009;89((1):27-36.

11. Coivov, Volpi R, Caprettil, Cafferi G. Effects of Pyridostigmine and Naloxane on the abnormal TSH response to TRH during starvation in humans. Alcohol Clin Express.2009;21(7):1308-11.

12. Baumgartner A, Gra "f K-J, Ku“ rten I \& Meinhold H. The hypothalamic-pituitary- thyroid axis in psychiatric patients and healthy subjects: Parts 1-4. Psychiatry Research. 1988; 24:271-332.

13. Faber J. The metabolism of iodothyronines in health and disease with special reference to diiodothyronines. Danish Medical Bulletin.2008;(31): 257-270.

14. Sabeen Aatif, Rana Qamar, Iftekhar Ahmad, Khalid Imran. Sick Euthyroid syndrome:Thyroid function abnormalities in patients with non-Thyroid illness. JLUMHS.83-86.

15. H. D'haenen, J. A. D. Boer, and P. Willner, Biological Psychiatry, vol. 1, Wiley, Chichester, UK, 2002.

16. 0. M. Wolkowitz and A. J. Rothschild, Psychoneuroendocrinology: The Scientific Basis of Clinical Practice, American Psychiatric, Washington, DC, USA, 1st edition, 2003.

17. B. N. Premachandra, M. A. Kabir, and I. K. Williams, "Low T3 syndrome in psychiatric depression. JEl.2006; 29(6):568-572.

18. Ganang WF. The thyroid gland. In: Ganong WF (ed). Review of Medical Physiology, $22^{\text {nd }} E d n$. The McGrawHill Companies. Inc.2005; 317-3.

19. Anderson S, Pederson KM, Bruun NH, Laurberg P. Narrow individual variations in serum T4 and T3 in normal subjects: a clue to the understanding of sub clinical thyroid disease. J Clin Endocrinol Metab. 2002;87:1068-72. 


\section{AUTHOR (S)}

1. DR. JAHANGIR SULTAN

Department of Pathology,

Chemical Section Continental Medical College,

Lahore, Pakistan

2. DR. TARIQ AFZAL

Department of Pathology,

Assistant Professor Sargodha Medical College,

Sargodha, Pakistan

3. DR. KOKAB JABEEN

Department of Pathology,

Continental Medical College, Lahore.

\section{Correspondence Address:}

\section{Dr. Jahangir Sultan}

Department of Pathology,

Chemical Section Continental Medical College,

Lahore, Pakistan

sultanjahangir8@gmail.com
Article received on: 28/03/2013 Accepted for Publication: 29/04/2013 Received after proof reading: $21 / 05 / 2013$

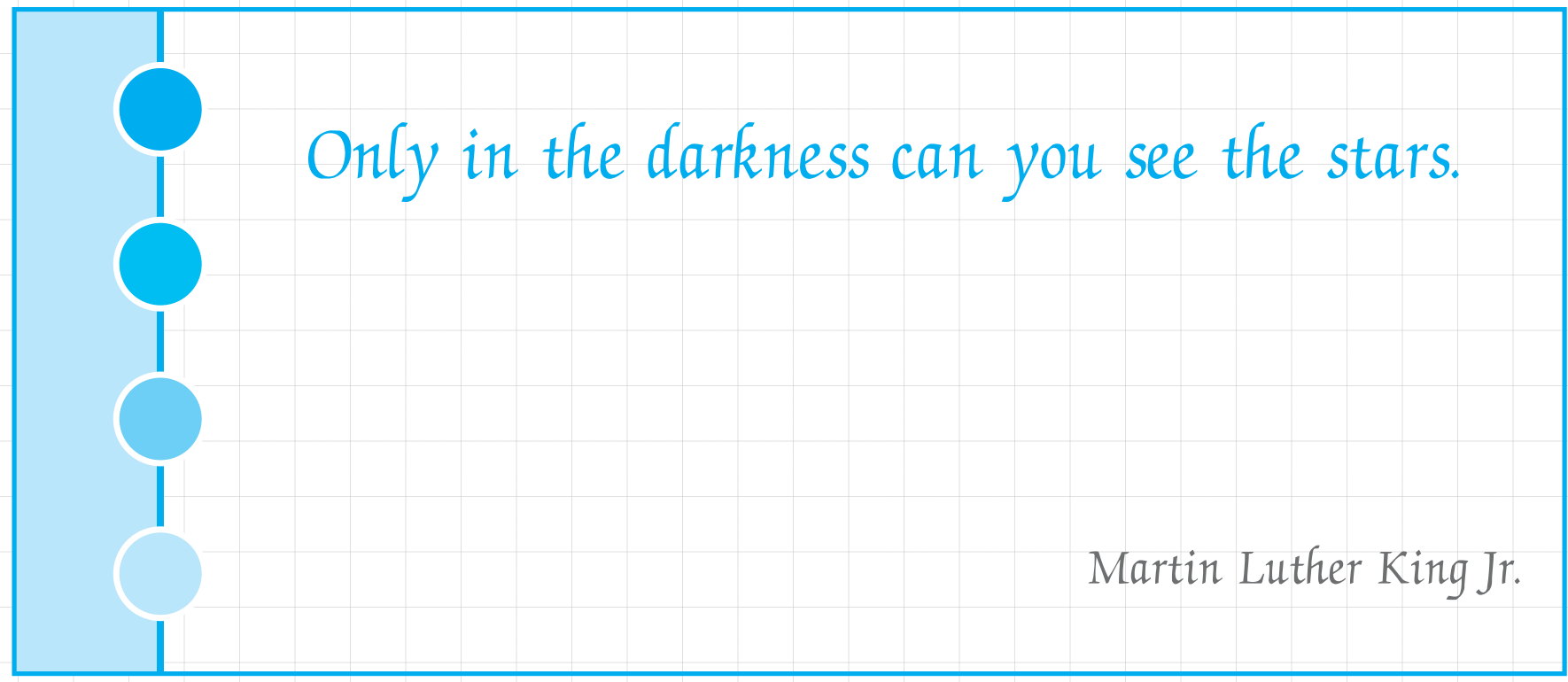

